\title{
Formación del profesorado ecuatoriano en autismo y asperger
}

\author{
Training of Ecuadorian teachers in Autism and Asperger \\ Formação de professores equatorianos em autismo e asperger
}

Johana Lourdes Narváez Intriago

Pontificia Universidad Católica del Ecuador, Ecuador

nij1@pucesd.edu.ec

https://orcid.org/0000-0003-1478-4435

Fernando Lara Lara

Pontificia Universidad Católica del Ecuador, Ecuador

IIf@pucesd.edu.ec

https://orcid.org/0000-0003-1545-9132

\section{Resumen}

El presente trabajo pretende relatar la experiencia de formación que supuso la implementación de un módulo de sensibilización para la atención de estudiantes con trastorno generalizado del desarrollo (autismo y asperger) dirigido al profesorado de educación básica media y elemental de una institución educativa en Santo Domingo (Ecuador). En el módulo se plantearon los siguientes temas principales: conceptualización, diagnóstico, características y estrategias para atender a estudiantes diagnosticados con autismo y asperger. La investigación fue de corte cualitativo, aunque también se utilizaron técnicas cuantitativas para el análisis de datos, mientras que el diseño fue no experimental. Se aplicaron encuestas a 15 docentes de la educación básica media y elemental en dos momentos (pretest y postest) para conocer el impacto del referido módulo, denominado Atendiendo a la diversidad sin exclusión, el cual procuró fomentar una cultura inclusiva en la institución desde el reconocimiento de ese trastorno. Entre los resultados principales se pueden mencionar la 


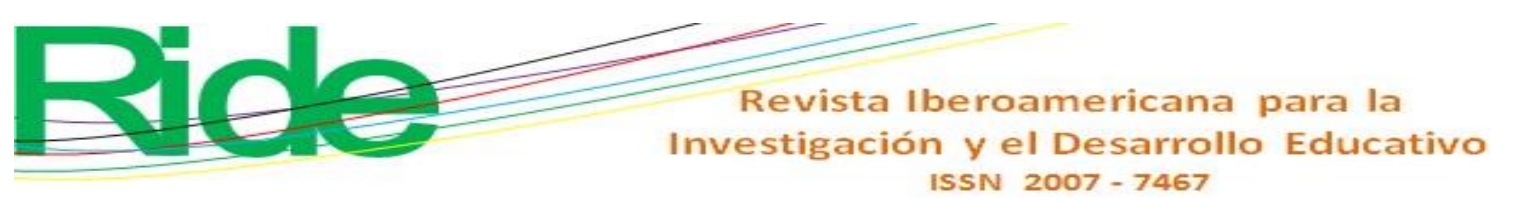

necesidad de formación docente actualizada sobre estrategias y metodologías de atención a estos estudiantes, así como la ausencia de una cultura inclusiva que reconozca los valores que aportan a la sociedad como consecuencia de una aparente cultura escolar asistencial. En cuanto a las conclusiones, se puede decir que en los participantes se reflejaron dificultades referidas a la identificación y diferenciación de los distintos trastornos, así como sobre el reconocimiento de las aportaciones que estas personas pueden realizar a la sociedad y a su grupo de iguales. En síntesis, se evidencia una predominante visión asistencial etnocéntrica e incompatible con una cultura educativa inclusiva.

Palabras clave: cultura, educación, evaluación, exclusión social, juicio de valor, formación.

\section{Abstract}

This paper intends to relate the training experience involved in the implementation of a sensitization module for the attention of students with Generalized Developmental Disorder (Autism and Asperger) aimed at teachers of elementary and elementary basic education, of an Educative Institute in Santo Domingo de los Tsáchilas (Ecuador). In the module they are raised as main themes: conceptualization, diagnosis, characteristics and strategies to work with Austism and Asperger' students diagnosed with Autism and Asperger. A qualitative investigation is carried out in which quantitative techniques were used for data analysis, with a non-experimental design; Surveys were applied to 15 teachers of elementary and basic education, in two moments (pretest and posttest) in relation to implement an Attending to Diversity without exclusion module with the aim of fostering an inclusive culture in the center since the recognition of this Disorder. Among the main results we can mention: the need for up-to-date teacher training on strategies and methodologies of attention to these students, and the absence of an inclusive culture that recognizes the values they contribute to society as a result of an apparent school attendance culture; In the same way, among the essential conclusions to mention: difficulties were identified and also differentiate many disorders, and in the recognition of the contributions that these people can make to society and its peer group, colliding in a predominant care vision ethnocentric, incompatible with an inclusive educational culture.

Keywords: culture, education, evaluation, social exclusion, value judgment, training. 


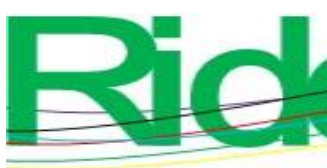

Revista Iberoamericana para la
Investigación y el Desarrollo Educativo ISSN $2007-7467$

\section{Resumo}

O presente trabalho tem como objetivo relatar a experiência de formação que envolveu a implementação de um módulo de conscientização para o atendimento de alunos com transtorno invasivo do desenvolvimento (autismo e Asperger) dirigido a professores do ensino fundamental e médio de uma instituição de ensino em Santo Domingo (Equador). No módulo foram levantados os seguintes tópicos principais: conceituação, diagnóstico, características e estratégias para atender alunos com diagnóstico de autismo e asperger. A pesquisa foi qualitativa, embora técnicas quantitativas também tenham sido utilizadas para a análise dos dados, enquanto o delineamento foi não experimental. Foram aplicadas pesquisas a 15 professores do ensino fundamental e médio em dois momentos (pré-teste e pós-teste) para conhecer as repercussões do referido módulo, denominado Atendendo à diversidade sem exclusão, que buscou promover uma cultura inclusiva na instituição a partir do reconhecimento daquele transtorno. Dentre os principais resultados, podemos citar a necessidade de uma formação docente atualizada sobre estratégias e metodologias de atenção a esses alunos, bem como a ausência de uma cultura inclusiva que reconheça os valores que eles contribuem para a sociedade em decorrência de uma aparente cultura de atendimento escolar. Quanto às conclusões, pode-se dizer que os participantes refletiram dificuldades relacionadas à identificação e diferenciação dos diferentes transtornos, bem como ao reconhecimento das contribuições que essas pessoas podem dar à sociedade e ao seu grupo de pares. Em resumo, uma visão etnocêntrica predominante do cuidado é evidente e incompatível com uma cultura educacional inclusiva.

Palavras-chave: cultura, educação, avaliação, exclusão social, juízo de valor, treinamento. Fecha Recepción: Marzo 2020 Fecha Aceptación: Enero 2021 


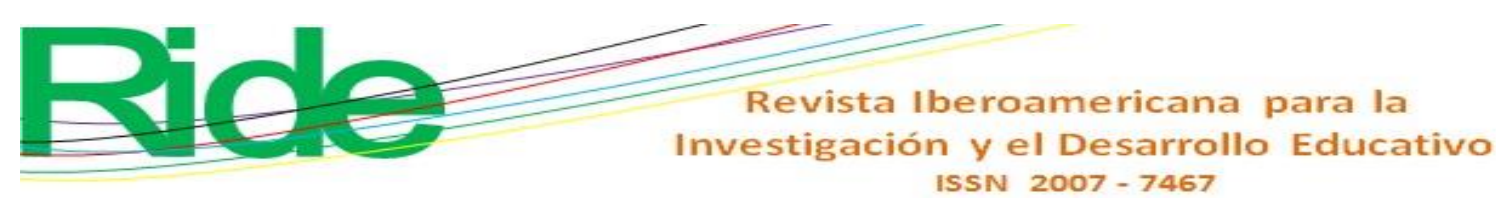

\section{Introducción}

La actualidad de la educación inclusiva requiere de una atención personalizada que demanda recursos humanos capacitados en formación y evaluación. En tal sentido, la realidad del autismo y asperger es una de las tareas que debe ser afrontada por cualquier política de educación inclusiva (Beltrán, Martínez y Vargas, 2015; Durán y Giné, 2011), con una relevancia jurídica en Ecuador constatable en la medida que se aspira a una sociedad del buen vivir (Lara y Gestner, 2016; Lara y Jaramillo, 2019; Villagómez y Cunha de Campos, 2014).

En tal sentido, el derecho a la educación se encuentra consagrado en la Declaración de los Derechos Humanos de las Naciones Unidas, pero su vigencia necesita de una continua discusión y reflexión sobre su implementación en la línea que plantean los 17 objetivos de desarrollo sostenible (ODS) propuestos por el Programa de la Naciones Unidas para el Desarrollo. De hecho, en el cuarto de dichos objetivos se hace referencia a la educación de calidad, pues para el año 2030 se procura "garantizar una educación inclusiva y equitativa de calidad y promover oportunidades de aprendizaje permanente para todos" (ODS, 2017, p. 7), es decir, la comunidad educativa conformada por padres de familia, instituciones, profesorado, medios de comunicación y sociedad en general.

En esta línea, una propuesta de enfoque crítico es la que proporciona la educación inclusiva — como mencionan Correa Montoya y Rúa (2018) — en la cual se señala que "el entorno educativo es el que debe ofrecer una respuesta diferenciada y adaptarse a las necesidades educativas de las personas, dentro del sistema educativo regular y en compañía de los maestros y de otros estudiantes con y sin discapacidad" (p. 100).

En Ecuador, el Ministerio de Educación Ecuatoriano (desde ahora Mineduc) en el 2017 aprobó el acuerdo número MINEDUC-MINEDUC-2017-00022-A con el fin de abordar la inclusión como horizonte educativo. De ese modo, quedó establecido en el literal $o$ una de las obligaciones del Estado, es decir, "elaborar y ejecutar las adaptaciones curriculares necesarias para garantizar la inclusión y permanencia dentro del sistema educativo de las personas con discapacidades, adolescentes y jóvenes embarazadas" (p. 1); sin embargo, este desafío supone la apuesta de un proceso de muda cultural que permita la incorporación de la participación de la diversidad que ofrece cada persona. Con este afán, distintos autores (García y Hernández, 2016) apuestan por el fortalecimiento de este objetivo a través de la formación y la promoción de una cultura educativa inclusiva (Cruz Vadillo, 2018; Mogollón 


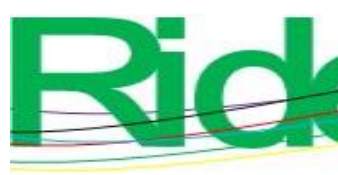

Revista Iberoamericana para la Investigación y el Desarrollo Educativo ISSN $2007-7467$

y Falla, 2014), de reconocimiento y atención de las realidades de las personas con autismo y asperger.

Para mayor abundamiento, el ordenamiento jurídico ecuatoriano plantea un escenario novedoso en cuanto a la educación (Córdova, Lara y García, 2017; Lara, 2019a, 2019b; Lara y De la Herrán, 2016; Lara, De la Herrán y Arévalo, 2019; Lara, Sousa, De la Herrán, Nieto, y Gerstner, 2017), pues se establece como modelo a seguir el que propone la educación inclusiva (De la Herrán, Ruiz y Lara, 2018), lo que ha supuesto un cambio importante en la cultura educativa del país (con la prudencia que requiere cualquier movimiento de cambio) y en la definición de sus horizontes próximos. Así por ejemplo, la Ley Orgánica de Educación Intercultural (2017) consagra en su artículo 47 que "tanto la educación formal como la no formal tomarán en cuenta las necesidades educativas especiales de las personas en lo afectivo, cognitivo y psicomotriz" (p. 40).

En Ecuador, la presencia de alumnos con estos trastornos de personalidad se halla en $0.28 \%$ de estudiantes menores de 5 años de edad, y $1.7 \%$ entre los 6 y los 14 años de edad (Ministerio de Salud Pública del Ecuador, 2017); así, se eleva a constante la preocupación sobre la formación y actualización docente en la atención de estos estudiantes, y en la posibilidad de revertir su experiencia en el aula y en el centro educativo. Sobre esta preocupación y su atención se ocupan distintos estudios realizados, como el de Viloria (2016) o Granada, Pómes y Sanhueza (2013), quienes afirman que "en general los profesores no se sienten competentes ni a gusto enseñando a niños con diversas NEE”' (p. 56).

Según el Manual diagnóstico y estadístico de los trastornos mentales (desde ahora DSM-IV) (American Psychologycal Association, 2014), el trastorno generalizado del desarrollo como (desde ahora TGD) constituye "una perturbación grave y generalizada de varias áreas del desarrollo: habilidades para la interacción social, habilidades para la comunicación o la presencia de comportamientos, intereses y actividades estereotipados" (p. 69); por otro lado, el TGD también es definido como una alteración grave y generalizada en diferentes áreas del desarrollo, tales como dificultad en el ámbito social, problemas de comunicación (comprensión del lenguaje verbal y no verbal), conductas repetitivas y escasa flexibilidad mental (Aguirre, Álvares, Angulo y Prieto, 2014).

Las personas con autismo manifiestan alteraciones en algunas de sus cualidades psicológicas, ya sean afectivas, de comunicación o motrices. Los investigadores Chicon, Oliveira, Garozzi, Coelho y Carvalho (2019) expresan que "estudos têm revelado Que o 


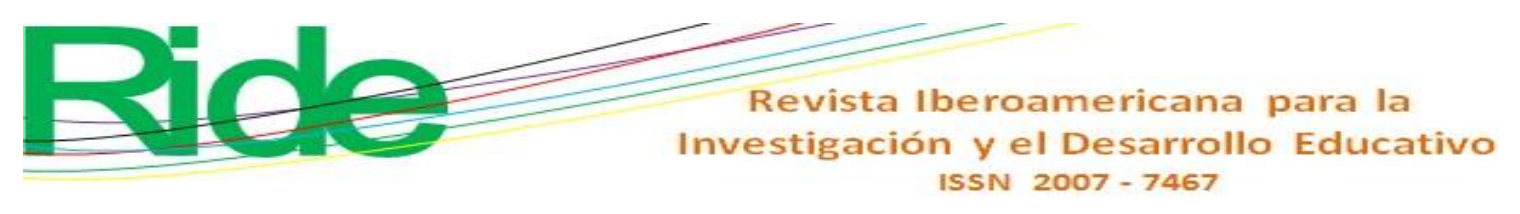

Autismo é compreendido Como uma síndrome comportamental e caracterizado por déficit na interac AO sociales, na linguagem e nas Alterac OES de comportamento" (p. 170). Como complemento a la definición anterior, el Instituto de Evaluación Tecnológica en Salud (2015) lo define como "una disfunción neurológica crónica con fuerte base genética que desde edades tempranas se manifiesta en una serie de síntomas basados en la tríada de Wing que incluye: la comunicación, flexibilidad e imaginación e interacción social” (p. 29). El autismo, por tanto, es uno de los trastornos más complejos y serios del desarrollo, pues presenta características que afectan las áreas sociales y de aprendizaje del individuo:

Has been characterized by a lack of understanding and a limited production of implicit meanings in conversation; in other words, people with Autismo have particular weaknesses in areas of non-literal meaning (e.g., figurative or metaphorical expressions, humour, irony or sarcasm) and tend to use words and expressions literally (Rodríguez, 2019, p. 54).

Por otra parte, el asperger es una derivación del autismo con ciertas similitudes, aunque también diferencias: una de ellas es que las personas con autismo presentan dificultades en el lenguaje, a diferencia de los estudiantes con asperger. La American Psychiatric Association (citada por Vives y Ascanio, 2012) lo define como "un trastorno generalizado del desarrollo caracterizado por una alteración cualitativa de la interacción social, por la presencia de intereses restrictivos y de comportamientos estereotipados" (p. 402). Las personas con síndrome de Asperger (desde ahora SA) presentan características muy notorias en cuanto su personalidad, como son la dificultad en las funciones ejecutivas, patrones de habla repetitivos, problemas de relaciones de iguales, inflexibilidad mental y comportamental, alteración de la expresión emocional y motora entre otros (Mineduc, 2016).

El asperger y el autismo (desde ahora TEA) son tratados en numerosos casos como trastornos similares; sin embargo, existen diferencias sustanciales relacionas con la motricidad gruesa, la coordinación motora y la psicomotricidad fina, características que se encuentran presentes en las personas con asperger, pero que son difíciles de hallar en pacientes con autismo (Pérez y Martínez, 2014). Las personas con trastorno generalizado del desarrollo (autismo y asperger) si bien tienen singularidades como las descritas, poseen capacidades imprescindibles que deben ser valoradas de manera correcta para que puedan convertirse en fortalezas para estos individuos. 


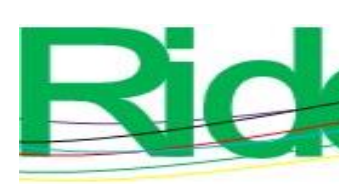
Revista Iberoamericana para la
Investigación y el Desarrollo Educativo
ISSN $2007-7467$

\section{Materiales y métodos}

Se realizó una investigación de corte cualitativo en la que se utilizaron técnicas cuantitativas para el análisis de datos. El diseño fue no experimental. La investigación de tipo explicativa estuvo dirigida a responder las causas y fenómenos físicos o sociales, mientras que la descriptiva se enfocó en los acontecimientos para luego organizarlos, tabularlos y describirlos (Abreu, 2013); finalmente, para la evaluación de la información y de los datos recabados se realizó un análisis estadístico mediante el programa Excel.

Asimismo, se aplicó una encuesta en dos momentos diferenciados: 1) previo a la realización del módulo sobre el TGD incluido en la capacitación — denominada Atendiendo a la diversidad sin exclusión, que intentó fomentar una cultura inclusiva en el centro educativo desde el reconocimiento de este trastorno-- y 2) luego de desarrollar el referido módulo. La encuesta fue elaborada atendiendo a las principales deficiencias mostradas en la evaluación inicial, y fue validada por expertos.

La población la conformaron los 40 docentes de una unidad educativa particular de Santo Domingo de los Tsáchilas (Ecuador). Esta institución oferta los siguientes niveles de educación: inicial, preparatoria, básica elemental y media y bachillerato general unificado. La muestra - compuesta por 15 docentes - fue no probabilística, pues el subnivel de educación con el que se trabajó fue elegido intencionalmente (Otzen y Manterola, 2017). Para la recolección de información y datos se aplicó una encuesta dirigida a los 15 docentes de los subniveles elemental y básica media como se puede visualizar en la tabla 1, con $73 \%$ de mujeres y $27 \%$ de hombres; el $40 \%$ tiene edades comprendidas entre los 18 y los 27 años, y $60 \%$ se encuentra en edades de 28 a 37 años; finalmente, en cuanto a su formación, $93 \%$ de los docentes tienen estudios de tercer nivel pertenecientes al área de la educación. 


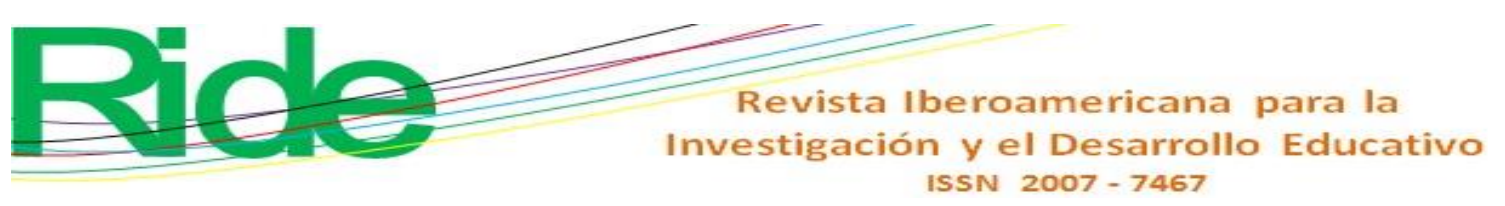

Tabla 1. Datos informativos de los participantes

\begin{tabular}{|l|c|c|}
\hline \multirow{4}{*}{ Edad } & Entre 18-27 años & $6(40 \%)$ \\
\cline { 2 - 3 } & Entre 28-37 años & $9(60 \%)$ \\
\cline { 2 - 3 } & Entre 38-47 años & $0(0 \%)$ \\
\cline { 2 - 3 } Sexo & Masculino & $4(27 \%)$ \\
\hline \multirow{3}{*}{$\begin{array}{l}\text { Años de } \\
\text { experiencia }\end{array}$} & Femenino & $11(73 \%)$ \\
\cline { 2 - 3 } & Menos de 1 año & $10(67 \%)$ \\
\cline { 2 - 3 } & $1-5$ años & $3(20 \%)$ \\
\hline \multirow{2}{*}{$\begin{array}{l}\text { Nivel de } \\
\text { educación }\end{array}$} & Tercer nivel & $2(13 \%)$ \\
\hline \multirow{2}{*}{\begin{tabular}{l} 
Área de formación \\
\cline { 2 - 3 }
\end{tabular}} & Cuarto nivel & $14(93 \%)$ \\
\cline { 2 - 3 } & Educación & $1(7 \%)$ \\
\hline
\end{tabular}

Fuente: Elaboración propia

\section{Resultados}

Los datos recabados antes y después de la implementación del módulo de formación evidenció rasgos generales (con la prudencia que requiere este análisis en el corto plazo) en cuanto al conocimiento de las conceptualización del trastorno del autismo y del asperger, comprensión de la naturaleza de sus necesidades, así como de las estrategias de enseñanza y adaptaciones curriculares, y de la disposición y reconocimiento de los valores que pueden aportar a la sociedad.

En cuanto a la conceptualización de este trastorno, se pudo comprobar que para aproximadamente la mitad de los docentes no eran familiares las definiciones proporcionadas por el DSM-IV (2014): es decir, TGD (53\%), TEA (47 \%) y SA (60 \%). Sin embargo, una vez implementada la formación planteada, se puedo obtener una mejoría de $13 \%$ (TGD), 27 $\%$ (TEA) y $20 \%$ (SA) (ver tabla 2). 
Tabla 2. Resultados del pretest y postest: conceptualización

\begin{tabular}{|l|c|c|c|c|c|c|c|c|c|}
\hline \multirow{2}{*}{ PREGUNTAS } & \multicolumn{3}{|c|}{ PRETEST } & \multicolumn{3}{c|}{ POSTEST } & \\
\cline { 2 - 10 } & $\begin{array}{l}\text { FRECU } \\
\text { ENCIA }\end{array}$ & $\begin{array}{c}\text { PORCENT } \\
\text { AJE }\end{array}$ & $\begin{array}{c}\text { FRECU } \\
\text { ENCIA }\end{array}$ & $\begin{array}{c}\text { PORCENT } \\
\text { AJE }\end{array}$ & $\begin{array}{c}\text { PORCENT } \\
\text { AJE DE } \\
\text { AVANCE }\end{array}$ \\
\cline { 2 - 10 } & C & I & C & I & C & I & C & I & $\%$ \\
\hline Concepto del TGD & 8 & 7 & $53 \%$ & $47 \%$ & 10 & 5 & $67 \%$ & $\begin{array}{c}33 \\
\%\end{array}$ & $13 \%$ \\
\hline Concepto del TEA & 7 & 8 & $47 \%$ & $53 \%$ & 11 & 4 & $73 \%$ & $\begin{array}{c}27 \\
\%\end{array}$ & $27 \%$ \\
\hline Concepto SA & 9 & 6 & $60 \%$ & $40 \%$ & 12 & 3 & $80 \%$ & $\begin{array}{c}20 \\
\%\end{array}$ & $20 \%$ \\
\hline
\end{tabular}

Fuente: Elaboración propia

C: respuesta correcta

I: respuesta incorrecta

Otro aspecto evaluado fue la caracterización del autismo y asperger, pues después de tabular el pretest (ver tabla 3) se determinó que más de $50 \%$ de los docentes evaluados desconocen temáticas vinculadas con la identificación de las características generales y específicas de estos trastornos. Sin embargo, en el momento posterior a la aplicación del módulo de sensibilización se obtuvo $27 \%$ de mejora en cuanto a su reconocimiento, lo que demuestra mayor dificultad en las características específicas del SA con un avance limitado.

Tabla 3. Resultados del pretest y postest: características del TEA y SA

\begin{tabular}{|l|c|c|c|c|c|c|c|c|c|}
\hline \multicolumn{1}{|c|}{ PREGUNTAS } & \multicolumn{5}{c|}{ PRETEST } & \multicolumn{3}{c|}{ POSTEST } & \\
\cline { 2 - 11 } & \multicolumn{2}{|c|}{$\begin{array}{c}\text { FRECU } \\
\text { ENCIA }\end{array}$} & $\begin{array}{l}\text { PORCENT } \\
\text { AJE }\end{array}$ & $\begin{array}{l}\text { FRECU } \\
\text { ENCIA }\end{array}$ & $\begin{array}{c}\text { PORCENT } \\
\text { AJE }\end{array}$ & $\begin{array}{c}\text { PORCENT } \\
\text { AJE DE } \\
\text { AVANCE }\end{array}$ \\
\cline { 2 - 11 } & C & I & C & I & C & I & C & I & $\%$ \\
\hline $\begin{array}{l}\text { Características que } \\
\text { corresponden al SA }\end{array}$ & 7 & 8 & $47 \%$ & $53 \%$ & 11 & 4 & $73 \%$ & $\begin{array}{c}27 \\
\%\end{array}$ & $27 \%$ \\
\hline $\begin{array}{l}\text { Características que } \\
\text { corresponden al TEA }\end{array}$ & 6 & 9 & $40 \%$ & $60 \%$ & 10 & 5 & $67 \%$ & $\begin{array}{c}33 \\
\%\end{array}$ \\
\hline $\begin{array}{l}\text { Características que } \\
\text { específicas } \\
\text { corresponden al TEA }\end{array}$ & 8 & 7 & $53 \%$ & $47 \%$ & 12 & 3 & $80 \%$ & $\begin{array}{c}20 \\
\%\end{array}$ \\
\hline $\begin{array}{l}\text { Características que } \\
\text { específicas } \\
\text { corresponden al SA }\end{array}$ & 8 & 7 & $53 \%$ & $47 \%$ & 9 & 6 & $60 \%$ & $\begin{array}{c}40 \\
\%\end{array}$ \\
\hline
\end{tabular}




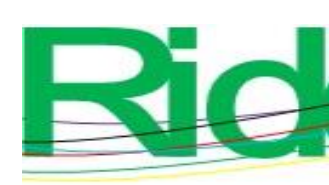

\section{Revista Iberoamericana para la Investigación y el Desarrollo Educativo ISSN 2007 - 7467}

Fuente: Elaboración propia

Por otra parte, en el pretest se evaluó parte de los conocimientos de los profesores acerca de las adaptaciones curriculares y estrategias metodológicas para relacionarse con estudiantes con SA y TEA (ver tabla 4). Al respecto, se obtuvo un porcentaje de respuestas incorrectas de $80 \%, 73 \%$ y $80 \%$, respectivamente. Luego de la aplicación se obtuvieron mejoras de $40 \%, 33 \%$ y $47 \%$ sobre esta temática.

Tabla 4. Resultados del pretest y postest: adaptaciones y estrategias

\begin{tabular}{|l|c|c|c|c|c|c|c|c|c|}
\hline \multirow{2}{*}{ PREGUNTAS } & \multicolumn{5}{c|}{ PRETEST } & \multicolumn{3}{c|}{ POSTEST } & \\
\cline { 2 - 9 } & $\begin{array}{l}\text { FRECU } \\
\text { ENCIA }\end{array}$ & $\begin{array}{c}\text { PORCENT } \\
\text { AJE }\end{array}$ & $\begin{array}{c}\text { FRECU } \\
\text { ENCIA }\end{array}$ & $\begin{array}{c}\text { PORCENT } \\
\text { AJE }\end{array}$ & $\begin{array}{c}\text { PORCENT } \\
\text { AJE DE } \\
\text { AVANCE }\end{array}$ \\
\cline { 2 - 10 } & C & I & C & I & C & I & C & I & $\%$ \\
\hline $\begin{array}{l}\text { Adaptaciones } \\
\text { curriculares: TEA y SA }\end{array}$ & 3 & 12 & $20 \%$ & $80 \%$ & 9 & 6 & $60 \%$ & 40 \\
$\%$ & $40 \%$ \\
\hline $\begin{array}{l}\text { Estrategias } \\
\text { metodológicas para } \\
\text { trabajar con estudiantes } \\
\text { con SA }\end{array}$ & 4 & 11 & $27 \%$ & $73 \%$ & 9 & 6 & $60 \%$ & 40 & $33 \%$ \\
\hline $\begin{array}{l}\text { Estrategias } \\
\text { metodológicas para } \\
\text { trabajar con estudiantes } \\
\text { con TEA }\end{array}$ & 3 & 12 & $20 \%$ & $80 \%$ & 10 & 5 & $67 \%$ & $\begin{array}{c}33 \\
\%\end{array}$ \\
\hline
\end{tabular}

Fuente: Elaboración propia

Finalmente, se evaluó a los docentes sobre la percepción hacia los valores que los estudiantes con autismo y asperger pueden aportar a la sociedad, y sobre la identificación de esos valores; en el primero caso, se obtuvieron porcentajes correctos altos en la primera pregunta (93\%) destinada a la percepción positiva de estos estudiantes en las instituciones educativas; sin embargo, en la segunda cuestión que se les planteó acerca de su reconocimiento, en gran su mayoría no consiguieron identificarlos, con solo $27 \%$ de aciertos. Tras la implementación del módulo, se pudo percibir una mayor competencia de identificación, aunque moderada, con tan solo $33 \%$ (ver tabla 5). 


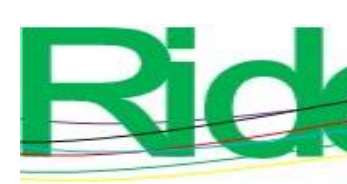

Revista Iberoamericana para la Investigación y el Desarrollo Educativo ISSN $2007-7467$

Otro aporte importante de este módulo se constató en el progreso del profesorado hacia el reconocimiento de estrategias metodológicas para atender a estudiantes con autismo y asperger, así como en la comprensión de que las adaptaciones curriculares deben responder en la mayoría de los casos a unos determinados ritmos, potencialidades y aptitudes definidos según las necesidades de estos alumnos, en lugar de la dosificación del contenido, como lo señalan Picardo et al. (2014). Finalmente, otro elemento vital fue el mejoramiento de los profesores en cuanto a la diferenciación consciente entre autismo y asperger.

\section{Conclusiones}

Luego de analizar los datos recabados se puede concluir que los participantes poseen un nivel bajo de conocimientos sobre estrategias para trabajar con estudiantes con autismo y asperger, pues se consiguió un rango elevado de respuestas incorrectas en la evaluación previa referida a $i$ ) si era necesario realizar adaptaciones curriculares a los estudiantes con autismo y asperger, $\mathrm{ii}$ ) mencionar tipos de estrategias metodológicas para trabajar con estos estudiantes, y iii) seleccionar estrategias adecuadas para trabajar con niños con autismo y asperger.

Con estos datos, se realizó una propuesta de intervención que consistió en la elaboración e implementación de un módulo diseñado no solo con el objetivo de capacitar a los profesores en relación con las realidades y la atención que requieren los estudiantes con TGD, sino también con el fin de mejorar la labor docente en el proceso de enseñanzaaprendizaje, y favorecer una actitud favorable y de aprecio a los valores que pueden aportar. En tal sentido, durante las cinco sesiones de intervención que se planificaron, los docentes mostraron una predisposición positiva hacia el módulo y los temas que se trataron, así como hacia las metodologías activas en el aula que se propusieron, como fue el aprendizaje cooperativo y el aula invertida.

De hecho, luego de la aplicación de la propuesta de intervención, se aplicó un postest con el objetivo de evaluar los conocimientos adquiridos por los participantes sobre la realidad del TGD. Los resultados fueron favorables, pues se detectó un incremento importante en los aciertos en las temáticas donde los docentes presentaban más carencias.

En resumen, si bien se aprecia la visión de una cultura educativa asistencial en la mayoría de los docentes, la formación del profesorado que se pudo realizar propuso una reflexión sobre la responsabilidad con estos estudiantes. 


\section{Contribuciones a futuras líneas de investigación}

Algunas líneas de investigación pueden ser las siguientes:

- Visibilizar las aportaciones que los distintos estudiantes con TGD pueden aportar a la unidad educativa y a su comunidad.

- Proponer capacitaciones sobre el ego docente y su relación con la formación del profesorado en educación inclusiva.

- Incorporar a los procesos de formación grupos como las familias, miembros de la administración y servicios, así como autoridades de la institución.

\section{Agradecimientos}

Este trabajo fue patrocinado por el proyecto de investigación y vinculación Presencia del postgrados de la PUCE Santo Domingo en la investigación y vinculación de su oferta académica (PIV-SANTODOMINGO-01-2020), perteneciente al grupo de investigación Educación, lengua, comunicación, política e innovación (GI-SANTO DOMINGO-19-2019). 


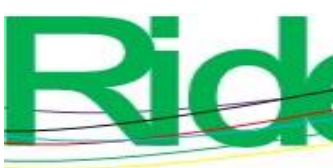

Revista Iberoamericana para la
Investigación y el Desarrollo Educativo
ISSN $2007-7467$

Larraceta, A. (2020). La formación del profesorado sobre el autismo basada en la evidencia: la instrucción e intervención mediada por pares. Revista Electrónica Interuniversitaria de Formación del Profesorado, 23(1), 127-142. Doi: https://doi.org/10.6018/reifop.393811

Ley Orgánica de Educación Intercultural (2017). Recuperado de https://educacion.gob.ec/wp-

content/uploads/downloads/2017/02/Ley_Organica_de_Educacion_Intercultural_L OEI_codificado.pdf

Mazzer, M. (2015). Inclusion of students with Autism Spectrum Disorders in Italian Schools: From Theory to Practice. Open Journal of Social Sciences, 3, 91-96.

Ministerio de Educación de Chile (2010). Manual de apoyo docente: educación de estudiantes que presentan trastorno del espectro de autismo. SDL Impresores.

Ministerio de Educación de Chile (2016). Guía de apoyo técnico-pedagógico: necesidades educativas especiales en nivel de educación patibularia asociada al autismo. Recuperado de http://especial.mineduc.cl/wpcontent/uploads/sites/31/2016/08/GuiaAutismo.pdf

Ministerio de Educación (2017). Acuerdo Nro. MINEDUC-MINEDUC-2017-00022-A. Recuperado de https://educacion.gob.ec/wpcontent/uploads/downloads/2017/04/MINEDUC-MINEDUC-2017-00022-A.pdf

Ministerio de Salud Pública del Ecuador [Mineduc] (2017). Trastornos del Espectro Autista en niños y adolescentes: detección, diagnóstico, tratamiento, rehabilitación y seguimiento. Recuperado de: https://www.salud.gob.ec/wpcontent/uploads/2014/05/GPC_Trastornos_del_espectro_autista_en_ninos_y_adoles centes-1.pdf

Mogollón, D. y Falla, B. (2014). La escuela inclusiva: desafíos y oportunidades del psicólogo escolar. Alteridad, 9(2), 92-107.

Objetivo de Desarrollo Sostenible [ODS] (2017). Desglosar el objetivo de desarrollo sostenible 4 de educación 2030.

Otzen, T. y Manterola, C. (2017). Técnicas de muestreo sobre una población a estudio. International Journal of Morphology, 35(1), 227-232.

Pérez, P. y Martínez, L. (2014). Perfiles cognoscitivos en el trastorno autista de alto funcionamiento y el síndrome de Asperger. CES Psicologia, 7(1), 141-155. 


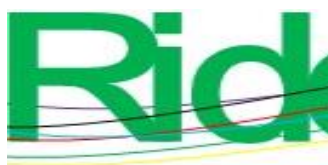

Revista Iberoamericana para la Investigación y el Desarrollo Educativo ISSN $2007-7467$

Picardo, O., Miranda, A., Escobar, J. y Olivia, H. (2014). Pedagogía, didáctica y autismo. UFG.

Rey, A. y García, B. (2018). Manual de estilo: cómo abordar el trastorno del espectro del autismo desde los medios de comunicación. Recuperado de http://www.euskadi.eus/dokumentazioa/2018/como-abordar-el-trastorno-delespectro-del-Autismo-desde-los-medios-de-comunicacion/web01-a2zesosa/eu/

Rodríguez, F. (2019). The construction of cooperative and inferential meaning by children with Asperger síndrome. Topics in Linguistics, 20(1), 54-67.

Villagómez, M. y Cunha de Campos, R. C. (2014). Buen vivir y educación para la práctica de la interculturalidad en el Ecuador. Otras prácticas pedagógicas son necesarias. Alteridad, 9(1), 35-42.

Viloria, M. (2016). Integración escolar del alumnado con necesidades educativas especiales en el municipio Caroní de Venezuela (tesis doctoral). Universitat de Girona, Venezuela.

Vives, C. y Ascanio, L. (2012). Resultados del tratamiento en un caso con trastorno de Asperger. Behavioral Psychology / Psicología Conductual, 20(2), 401-419.

Zambrano, R. y Orellana, M. (2018). Actitudes de los docentes hacia la inclusión escolar de niños con autismo. Killkana Sociales: Revista de Investigación Científica, 2(4), 3948. 


\begin{tabular}{|l|l|}
\hline Rol de Contribución & Autor (es) \\
\hline Conceptualización & $\begin{array}{l}\text { Johana Lourdes Narváez Intriago, Fernando Lara Lara } \\
\text { igual }\end{array}$ \\
\hline Metodología & $\begin{array}{l}\text { Johana Lourdes Narváez Intriago, Fernando Lara Lara } \\
\text { igual }\end{array}$ \\
\hline Software & Johana Lourdes Narváez Intriago \\
\hline Validación & Johana Lourdes Narváez Intriago \\
\hline Análisis Formal & Johana Lourdes Narváez Intriago \\
\hline Investigación & $\begin{array}{l}\text { Johana Lourdes Narváez Intriago, Fernando Lara Lara } \\
\text { igual }\end{array}$ \\
\hline Recursos & Johana Lourdes Narváez Intriago \\
\hline Curación de datos & Johana Lourdes Narváez Intriago \\
\hline $\begin{array}{l}\text { Escritura - Preparación del } \\
\text { borrador original }\end{array}$ & Johana Lourdes Narváez Intriago \\
\hline Escritura - Revisión y edición & Fernando Lara Lara \\
\hline Visualización & Fernando Lara Lara \\
\hline Supervisión & Fernando Lara Lara \\
\hline Administración de Proyectos & Johana Lourdes Narváez Intriago \\
\hline Adquisición de fondos & Johana Lourdes Narváez Intriago \\
\hline
\end{tabular}

